\title{
Temporal Profile of De Novo Development of Moyamoya Vasculopathy in an Adult -Case Report-
}

\author{
Yoshiteru SHIMODA, ${ }^{1}$ Miki FUJIMURA, ${ }^{1}$ Takashi InOUE, ${ }^{1}$ \\ Hiroaki SHIMIZU, ${ }^{1}$ and Teiji TOMINAGA ${ }^{2}$ \\ ${ }^{1}$ Department of Neurosurgery, Kohnan Hospital, Sendai, Miyagi; \\ ${ }^{2}$ Department of Neurosurgery, Tohoku University School of Medicine, Sendai, Miyagi
}

\begin{abstract}
A 46-year-old woman developed transient ischemic attack (TIA) caused by progressive moyamoya vasculopathy in the right hemisphere. She had presented with minor cerebellar infarction due to left vertebral artery dissection 7 years prior to the onset of TIA. Initial carotid angiography at the onset of vertebral artery dissection demonstrated absence of steno-occlusive changes in the anterior circulation. During the next 7 years, she had been strictly followed up with magnetic resonance angiography every 6 months, which demonstrated the asymptomatic development of steno-occlusive change in the proximal right middle cerebral artery, finally involving the terminal internal carotid artery. Carotid angiography at the onset of TIA showed terminal internal carotid artery stenosis with abnormal vascular network at the right base of the brain, indicating a definitive diagnosis of moyamoya vasculopathy with unilateral involvement. She underwent right superficial temporal artery-middle cerebral artery anastomosis without complication one month later. TIA completely disappeared after surgery, and no cerebrovascular events occurred during the follow-up period of 6 months. De novo formation of moyamoya vasculopathy is extremely rare in adulthood. The present case demonstrated the entire temporal profile of the development of this rare entity in adulthood. The previous presentation of vertebral artery dissection before the development of moyamoya vasculopathy as well as the initiation of steno-occlusive change at the middle cerebral artery is apparently unique.
\end{abstract}

Key words: moyamoya vasculopathy, de novo development, progression, vertebral artery dissection, magnetic resonance imaging

\section{Introduction}

Moyamoya disease is a chronic, occlusive cerebrovascular disease with unknown etiology characterized by bilateral steno-occlusive changes at the terminal portion of the internal carotid artery (ICA) and an abnormal vascular network at the base of the brain. ${ }^{21)}$ The occlusive lesions frequently progress or unilateral lesions become bilateral in pediatric patients, ${ }^{3,15,20)}$ but progression of the stenoocclusive lesion is thought to be quite rare in adult patients. ${ }^{3,15,20)}$ Recently, increasing evidence suggests that adult patients with moyamoya disease and unilateral moyamoya disease also have substantial risk for progression of the steno-occlusive lesion. , $6,7,9,11-13,18,19)$ Nevertheless, de novo development of moyamoya vasculopathy is extremely rare, only reported in one case, ${ }^{6)}$ and the entire temporal profile of the development of moyamoya vasculopathy has not been demonstrated.

Here we report an adult case of de novo moyamoya vasculopathy, in which sequential magnetic resonance (MR)

Received May 31, 2011; Accepted September 12, 2011 angiographic images were obtained during the gradual development of the moyamoya vasculopathy.

\section{Case Report}

A 46-year-old woman presented with transient ischemic attack (TIA) causing weakness in her left upper limb and sensory disturbance at the left angle of her mouth, due to progressive moyamoya vasculopathy in the right hemisphere. She had no past history of connective tissue disorders including Marfin syndrome and Ehlers-Danlos syndrome. She had suffered a minor stroke involving the left cerebellum due to left vertebral artery (VA) dissection 7 years prior to the onset of TIA. She did not have any risk factors for cerebrovascular disease, including hypertension, diabetes mellitus, and dyslipidemia. Initial digital subtraction angiography at the onset of VA dissection had demonstrated irregularity of the left VA, but bilateral carotid angiography did not show any abnormality in the anterior circulation (Fig. 1). She was treated conservatively and was discharged without neurological symptoms. She was then strictly followed up with MR angiography 


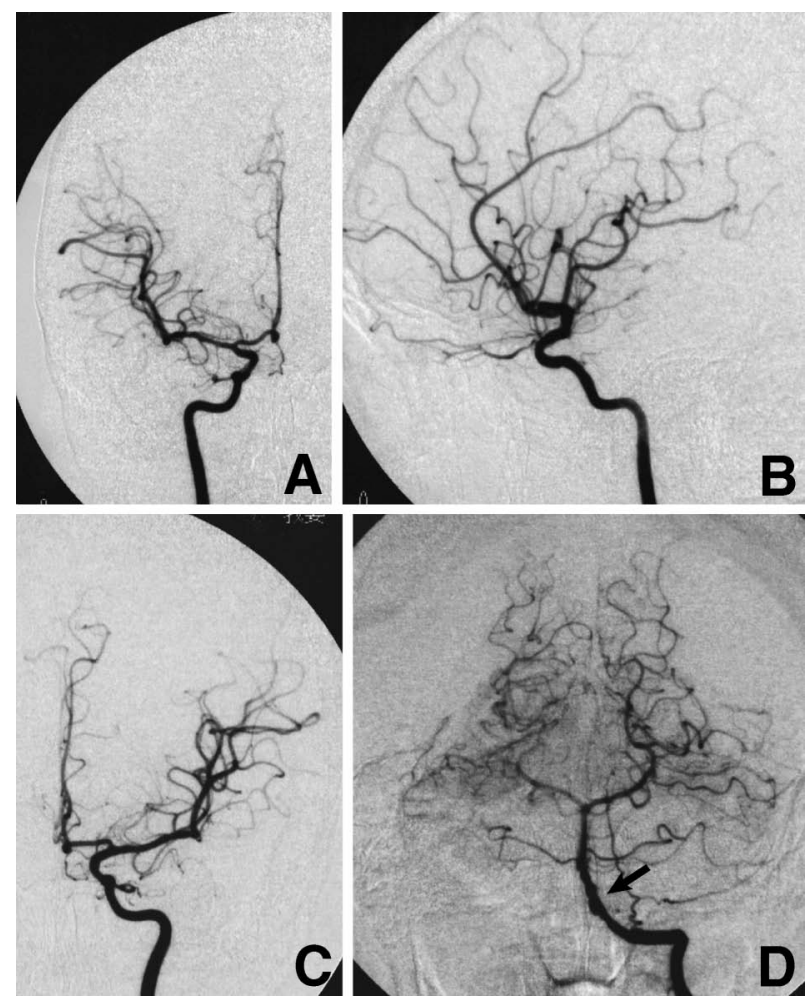

Fig. 1 Right internal carotid angiograms (A: anteroposterior view, B: lateral view), left internal carotid angiogram (C), and left vertebral angiogram (D) of the initial digital subtraction angiography at the onset of left vertebral artery dissection, demonstrating irregularity of the left vertebral artery $(D$, arrow), whereas no steno-occlusive lesions in the anterior circulation (A-C).
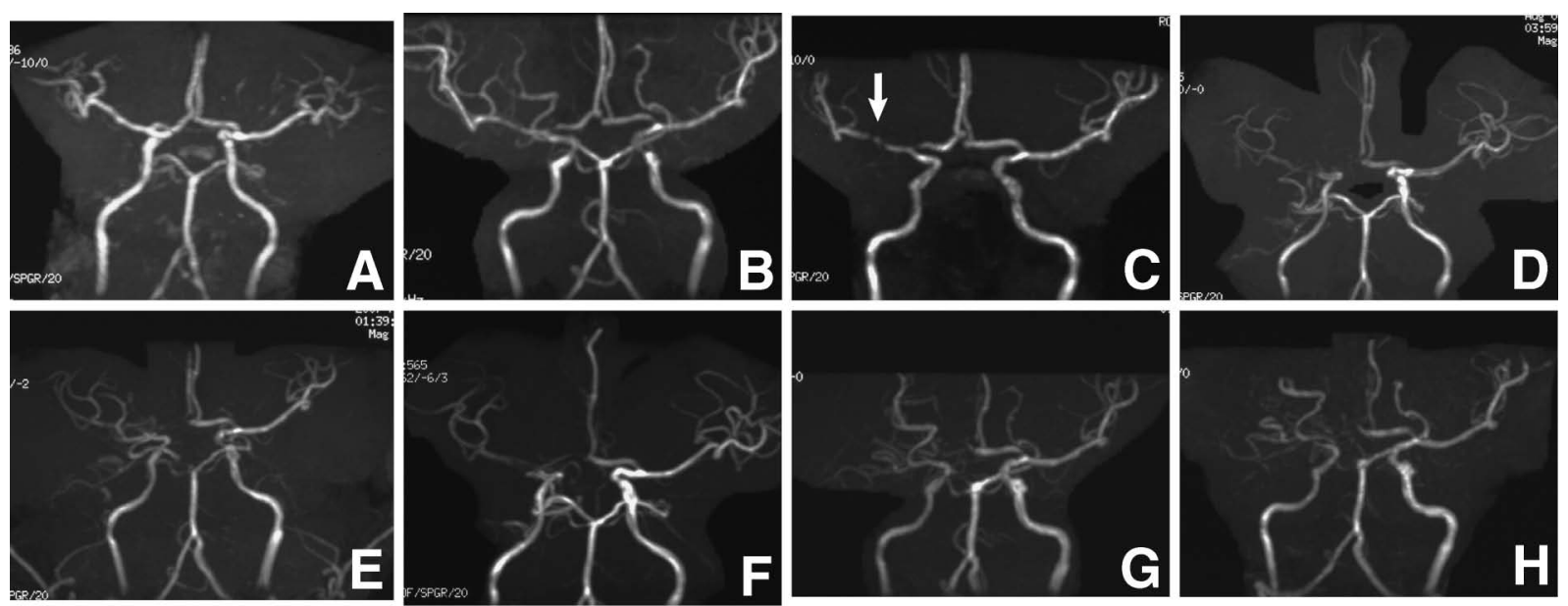

Fig. 2 Temporal profile of magnetic resonance angiography demonstrating the gradual development of steno-occlusive change that began at the proximal portion of right middle cerebral artery (arrow in C) and anterior cerebral artery (B, C), and finally involved the distal internal carotid artery (G, H). A: July 2003, B: December 2003, C: July 2004, D: August 2006, E: August 2007, F: August 2008, G: September 2009, H: June 2010. 

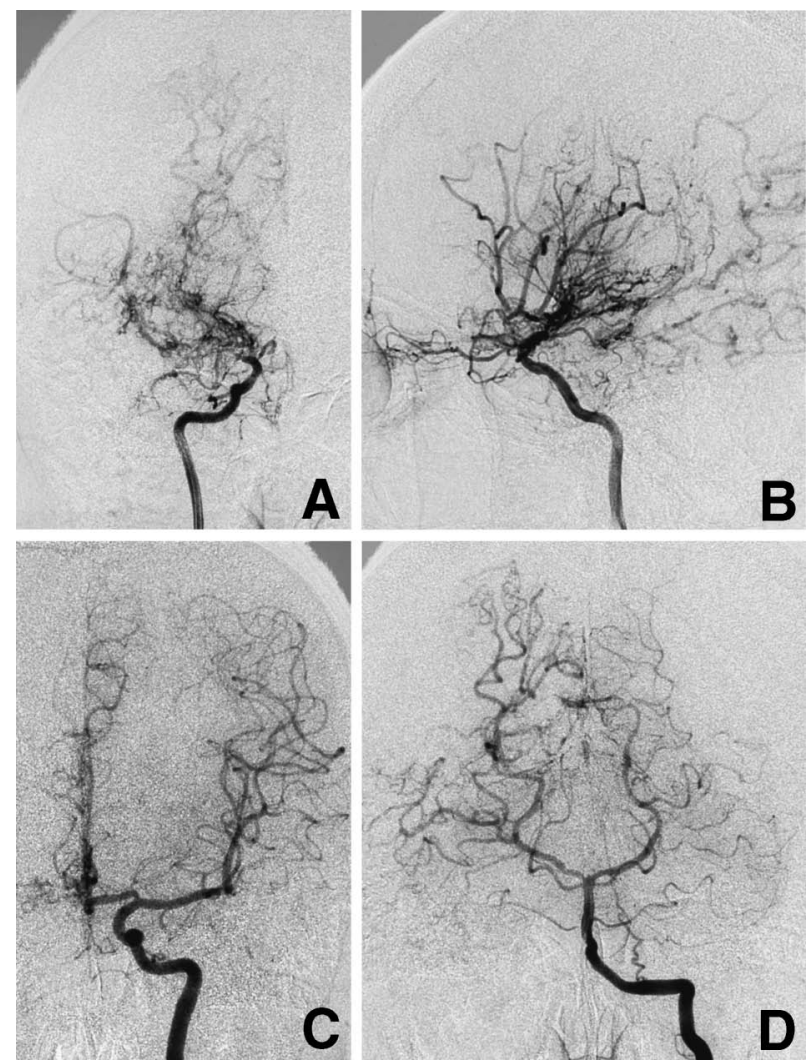

Fig. 3 Right internal carotid angiograms (A: anteroposterior view, B: lateral view), left internal carotid angiogram (C), and left vertebral angiogram (D) of preoperative digital subtraction angiography at the onset of transient ischemic attack (November 2010), demonstrating steno-occlusive changes at the terminal portion of the right internal carotid artery and an abnormal vascular network at the base of the brain.
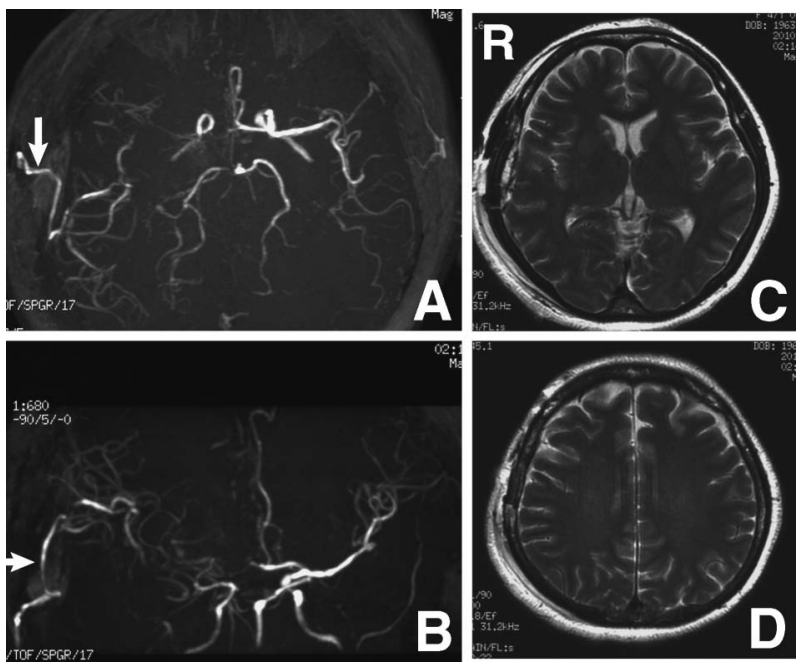

Fig. 4 Magnetic resonance (MR) angiograms $(A, B)$ and $T_{2^{-}}$ weighted MR images (C, D) after right revascularization surgery demonstrating apparently patent superficial temporal artery-middle cerebral artery bypass (arrows in A, B), and no signs of ischemic or hemorrhagic complications $(C, D)$. deterioration. Postoperative MR angiography showed the apparently patent STA-MCA bypass as a thick high signal intensity sign and MR imaging did not show any evidence of ischemic change (Fig. 4). TIA completely disappeared postoperatively, and the patient was discharged without neurological deficit 21 days after surgery. She suffered no further neurological events during the follow-up period of 6 months.

\section{Discussion}

The present case demonstrates the entire temporal profile of de novo development of moyamoya vasculopathy in a 46-year-old patient, who initially presented with VA dissection prior to the development of moyamoya vasculopathy. Repeated MR angiography allowed us to monitor the development and progression of steno-occlusive change in the MCA and anterior cerebral artery, and the formation of an abnormal vascular network at the base of the brain. The present case and the previously reported case of de novo development of moyamoya vasculopathy in adulthood ${ }^{6}$ show that moyamoya vasculopathy can newly develop even in adulthood.

Based on the general understanding of how moyamoya disease progresses, initiation of the steno-occlusive changes is considered to start from the terminal portion of the ICA. ${ }^{5,21)}$ Definitive diagnosis of moyamoya disease requires conventional angiography or MR angiography evidence of the involvement of the terminal ICA and the development of moyamoya vessels. ${ }^{5,21)}$ In a clinical setting, however, we sometimes encounter adult patients who present with unilateral moyamoya vasculopathy and contralateral MCA stenosis, which is distinct from this expected course of vasculopathy development and progression. ${ }^{2,9,19)}$ However, the exact temporal profile of the angioarchitecture of moyamoya vasculopathy from initiation to completion has remained unclear.

The present case clearly illustrates the detailed time course of the development and progression of moyamoya vasculopathy from initiation of the mid- $\mathrm{M}_{1}$ stenosis to the terminal ICA-involved stenosis with moyamoya vessel formation in a 46-year-old woman without any risk factors for atherosclerosis. These findings indicate that the origin and development of moyamoya vasculopathy could show more variations than previously understood. Early stage moyamoya vasculopathy might be considered in the differential diagnosis of patients without the risk factors for atherosclerosis who present with progressive disease involving the major artery around the terminal ICA. Alternatively, the possibility that the present case had a different underlying pathology from usual moyamoya disease cannot be excluded.

The underlying mechanism of such steno-occlusive changes has not yet been determined. Histological analysis of intracranial vessels in moyamoya disease has demonstrated subendothelial fibrous tissue occluding the vascular lumen, intimal thickening, smooth muscle cell proliferation, and the presence of macrophages and $\mathrm{T}$ cells. ${ }^{14,17)}$ Fragility of the medial layer is also thought to be one of the major pathologies of moyamoya disease. ${ }^{17)} \mathrm{A}$ re- 
cent report indicated the fragility of not only the ICA terminal but also the distal portion of MCA. ${ }^{23)}$ Moreover, patients with moyamoya disease often present with systemic arterial lesions including the STA or renal artery. ${ }^{1,22)}$ These findings strongly suggest that vascular abnormalities of moyamoya disease are not confined to the terminal ICA and surrounding areas, but are also distributed to other vessels distinct from the terminal ICA area. Interestingly, the occurrence of VA dissection preceded the development of the stenotic change in the MCA in the present case. This unique case demonstrated moyamoya vasculopathy complicated by VA dissection. Whether the association of VA dissection and subsequent moyamoya vasculopathy are coincidental or have a common pathology is unclear, but this rare association might be explained, at least in part, by the intrinsic vulnerability of intracranial vessels in patients with moyamoya vasculopathy. ${ }^{10,14,17,23)}$ Basic research exploring the mechanism underlying the vulnerability of moyamoya disease patients may be essential to address this important issue.

Surgical revascularization for moyamoya vasculopathy is thought to prevent cerebral ischemic attacks by improving cerebral blood flow, and STA-MCA anastomosis with or without indirect pial synangiosis is generally employed as a standard surgical procedure for moyamoya vasculopathy. ${ }^{4,8,16)}$ However, the natural course of this rare entity remains uncertain, so when the patient should undergo surgery during the clinical course has not yet been determined. A recent study based on retrospective data suggested that careful follow up of the asymptomatic hemisphere is an acceptable choice even in pediatric cases. ${ }^{15)}$ Our surgical indication includes the presence of ischemic symptoms, apparent hemodynamic compromise detected by SPECT, independent activity of daily living, and absence of major cerebral infarction. ${ }^{4)}$ We applied these principles in progressive cases, leading to favorable results. ${ }^{16)}$ In the present case, we performed revascularization surgery after the presentation of ischemic symptoms without perioperative complication. Therefore, we recommend strict follow up even for the asymptomatic hemisphere.

\section{References}

1) Aoyagi M, Fukai N, Yamamoto M, Matsushima $Y$, Yamamoto K: Early development of intimal thickening in superficial temporal arteries in patients with moyamoya disease. Stroke 27: 1750-1754, 1996

2) Choi HY, Lee JE, Jung YH, Cho HJ, Kim DJ, Heo JH: Progression of isolated middle cerebral artery stenosis into moyamoya disease. Neurology 68: 954, 2007

3) Ezura M, Yoshimoto T, Fujiwara S, Takahashi A, Shirane R, Mizoi K: Clinical and angiographic follow up of childhood onset moyamoya disease. Childs Nerv Syst 11: 591-594, 1995

4) Fujimura $M$, Shimizu $H$, Inoue $T$, Mugikura $S$, Saito $A$, Tominaga T: Significance of focal cerebral hyperperfusion as a cause of transient neurologic deterioration after EC-IC bypass for moyamoya disease: Comparative study with nonmoyamoya patients using ${ }^{123}$ I-IMP-SPECT. Neurosurgery 68: 957-965, 2011

5) Fukui M: Guidelines for the diagnosis and treatment of spontaneous occlusion of the circle of Willis ('moyamoya' dis- ease). Research Committee on Spontaneous Occlusion of the Circle of Willis (Moyamoya Disease) of the Ministry of Health and Welfare, Japan. Clin Neurol Neurosurg 99 Suppl 2: S238-240, 1997

6) Fukuya R, Yoshida K, Akiyama T, Kawase T: De novo development of moyamoya disease in an adult female. $J$ Neurosurg 111: 943-946, 2009

7) Houkin K, Yoshimoto T, Kuroda S, Ishikawa T, Takahashi A, Abe H: Angiographic analysis of moyamoya disease-how does moyamoya disease progress? Neurol Med Chir (Tokyo) 36: 783-788, 1996

8) Ishikawa T, Houkin K, Kamiyama H, Abe H: Effects of surgical revascularization on outcome of patients with pediatric moyamoya disease. Stroke 28: 1170-1173, 1997

9) Januschek E, Fujimura M, Mugikur S, Tominaga T: Progressive moyamoya syndrome associated with de novo formation of the ipsilateral venous and contralateral cavernous malformations: case report. Surg Neurol 69: 423-427, 2008

10) Kawagushi S, Sasaki T, Morimoto T, Kakizaki T, Kamada K: Characteristics of intracranial aneurysms associated with moyamoya disease. A review of 111 cases. Acta Neurochir (Wien) 138: 1287-1294, 1996

11) Kelly ME, Bell-Stephens T, Marks EM, Do HM, Steinberg GK: Progression of unilateral moyamoya disease: a clinical series. Cerebrovasc Dis 22: 109-115, 2006

12) Kuroda S, Hashimoto N, Yoshimoto T, Iwasaki Y: Radiological findings, clinical course, and outcome in asymptomatic moyamoya disease: results of multicenter survey in Japan. Stroke 38: 1430-1435, 2007

13) Kuroda S, Ishikawa T, Houkin K, Nanba R, Iwasaki Y: Incidence and clinical features of disease progression in adult moyamoya disease. Stroke 36: 2148-2153, 2005

14) Masuda J, Ogata J, Yutani G: Smooth muscle cell proliferation and localization of macrophages and T cells in the occlusive intracranial major arteries in moyamoya disease. Stroke 24: 1960-1967, 1993

15) Nagata $S$, Matsushima $T$, Morioka T, Matsuokado K, Mihara F, Sasaki T, Fukui M: Unilaterally symptomatic moyamoya disease in children: long term follow-up of 20 patients. Neurosurgery 59: 830-836, 2006

16) Narisawa A, Fujimura M, Tominaga T: Efficacy of the revascularization surgery for adult-onset moyamoya disease with the progression of cerebrovascular lesions. Clin Neurol Neurosurg 111: 123-126, 2009

17) Oka K, Yamashita M, Sadoshima S, Tanaka K: Cerebral haemorrhage in Moyamoya disease at autopsy. Virchows Arch Pathol Anat Histol 392: 247-261, 1981

18) Shirane R, Mikawa S, Ebina T: A case of adult moyamoya disease showing progressive angiopathy. Clin Neurol Neurosurg 101: 210-214, 1999

19) Smith ER, Scott RM: Progression of disease in unilateral moyamoya syndrome. Neurosurg Focus 24(2): E17, 2008

20) Soel HJ, Wang KC, Kim SK, Lee CS, Lee DS, Kim IO, Cho BK: Unilateral (probable) moyamoya disease: long term follow up of seven cases. Childs Nerv Syst 22: 145-150, 2006

21) Suzuki J, Takaku A: Cerebrovascular 'moyamoya' disease. Disease showing abnormal net-like vessels in base of brain. Arch Neurol 20: 288-299, 1969

22) Takagi Y, Hahsimoto N, Goto Y: Haemodynamic ischaemia in pediatric moyamoya disease associated with renovascular hypertension. Acta Neurochir (Wien) 139: 257-258, 1997

23) Takagi Y, Kikuta K, Kazuhiko N, Hashimoto N: Histological features of middle cerebral arteries from patients treated for moyamoya disease. Neurol Med Chir (Tokyo) 47: 1-4, 2007

Address reprint requests to: Miki Fujimura, MD, PhD, Department of Neurosurgery, Sendai Medical Center, 2-8-8 Miyagino, Miyagino-ku, Sendai, Miyagi 983-8520, Japan. e-mail: fujimur@nsg.med.tohoku.ac.jp 